\title{
José Garanger, un grand archéologue océaniste
}

Présentation du dossier d'hommage à José Garanger

Éric Conte et Christophe Sand

\section{(2) OpenEdition}

\section{Journals}

Édition électronique

URL : http://journals.openedition.org/jso/5840

DOI : $10.4000 /$ jso. 5840

ISSN : $1760-7256$

Éditeur

Société des océanistes

Édition imprimée

Date de publication : 30 juin 2009

Pagination : 5-10

ISBN : 978-2-85430-024-6

ISSN : 0300-953x

Référence électronique

Éric Conte et Christophe Sand, « José Garanger, un grand archéologue océaniste », Journal de la Société des Océanistes [En ligne], 128 | janvier-juin 2009, mis en ligne le 30 juin 2009, consulté le 22 septembre 2020. URL : http://journals.openedition.org/jso/5840 ; DOI : https://doi.org/10.4000/jso. 5840 


\section{Journal \\ de la \\ Société \\ des \\ Océanistes}

publié avec l'aide du CNRS et du CNL

Paris

128

Année 2009-1

C) Société des Océanistes 



\section{HOMMAGE À JOSÉ GARANGER}

SOUS LA DIRECTION DE

Éric ConTe et Christophe SAND 



\title{
José Garanger, un grand archéologue océaniste Présentation du dossier d'hommage à José Garanger
}

par

\author{
Éric CONTE* et Christophe SAND**
}

Comme il est de coutume pour un savant de renom, à l'occasion de son départ à la retraite, José Garanger reçut l'hommage de ses collègues et disciples sous la forme d'un bel ouvrage (Mémoire de pierre, Mémoire d'homme), témoin du respect et de l'affection de tous. Au début de cette publication (Julien et al., 1996), avait été rassemblée la bibliographie de José, comprenant pas moins de huit ouvrages et plus de quatrevingts articles scientifiques, témoins d'une carrière riche de résultats transmis sous différentes formes, avec en particulier trente articles « destinés à un public non spécialisé » (Julien et al., 1996 : 13-17). Lors de la réalisation de ce Festschrift, le choix des éditeurs, lié entre autres à des contraintes de place, avait nécessité de limiter les contributions aux collègues en poste, sans pouvoir intégrer la nouvelle génération de jeunes chercheurs qui émergeait au milieu des années 1990.

Dans ce numéro du Journal de la Société des Océanistes, c'est hélas à titre posthume qu'un hommage lui est à nouveau rendu. José nous a quittés le 26 décembre 2006. L'annonce de son départ a engendré un chagrin profond à travers le réseau étendu des archéologues et anthropologues océanistes répartis au quatre coins de la planète. Alors que José avait jusqu'au bout préféré le papier à lettres pour communiquer, le miracle d'internet a permis à trois générations d'Océanistes d'échanger durant les jours et les semaines suivantes, par-delà la distance entre
l'Europe, l'Amérique et le Pacifique, leur affection pour un collège et un " patron ». Les témoignages de sympathie ont afflué à Noisy-leGrand, juste reconnaissance de toute une profession pour un grand archéologue.

José Garanger a été le véritable pionnier de l'archéologie scientifique française dans la région (19871). Introduisant dans le Pacifique l'approche méthodologique de «l'école LeroiGourhan », faite de précision sur le terrain et de prudence dans les interprétations, son travail contrastait avec certaines pratiques en usage dans la région au début des années soixante. Pour l'avoir entendu évoquer les railleries de collègues de l'époque de le voir fouiller «à la petite cuillère », nous savons qu'au début de ses recherches en Polynésie, il dut faire preuve d'une certaine constance, étayée par des certitudes bien ancrées, pour persévérer dans des décapages minutieux de marae quand d'autres se livraient à une frénétique recherche d'objets... Les résultats acquis dans l'étude des monuments cérémoniels des îles de la Société comme des Tuamotu et, bien entendu, lors de la fouille des extraordinaires sites du Vanuatu (1965a, 1967a, 1972a), apportèrent la preuve sans appel de la pertinence de ses choix méthodologiques.

Ses recherches ont concerné autant la Mélanésie que la Polynésie et, dans ces deux régions, sa contribution scientifique fut majeure et conserve toute sa valeur. Sa thèse d'État sur les NouvellesHébrides (actuel Vanuatu) a exploré et révélé la

1. Tous les renvois bibliographiques sans autres précisions que la date concernent les ouvrages de José Garanger.

* Université de la Polynésie française, eric.conte@upf.pf

** Département Archéologie de Nouvelle-Calédonie, christophe.sand@gouv.nc 
préhistoire d'une région à l'époque largement inconnue des archéologues. Trente-cinq ans après leur publication (1972a, 1982), ses travaux demeurent une référence reconnue au niveau international pour cet archipel. En Polynésie orientale, ses recherches ont permis de mieux appréhender la variabilité autant structurelle que fonctionnelle des monuments religieux (marae) à Tahiti (1969a, 1969b) comme aux Tuamotu (Garanger, 1965b ; Garanger et Lavondès, 1967) et l'occupation par l'homme de plusieurs vallées peu connues de la presqu'île de Tahiti qu'il a étudiées dans des conditions matérielles très éprouvantes $(1964,1980 \mathrm{a})$.

Se gardant de spéculations purement théoriques, c'est dans ses travaux - par l'exemple en quelque sorte -, notamment à travers la célèbre découverte de la sépulture attribuée à Roy Mata, que José Garanger illustra la nouveauté et la fécondité d'une approche combinant les acquis de l'anthropologie et de l'archéologie (1972a, 1980b) qui devait inspirer bien des recherches dans le Pacifique et au-delà, trop nombreuses pour être énumérées ici.

Mais, outre les impératifs de la connaissance scientifique au plus haut niveau, le travail de José Garanger a revêtu une dimension humaine et culturelle annonçant des préoccupations très actuelles dans la communauté scientifique (Sand, 2003). Ses recherches ont été conduites en étroite collaboration avec les populations concernées et avec un profond respect de leurs valeurs (figure 1), même lorsque le travail de l'archéologue en était gêné, voire empêché. Il a aussi beaucoup œuvré pour la redécouverte et la réappropriation de leur passé par les Océaniens, un passé dont, dans certaines régions et notamment en Polynésie, ils étaient largement oublieux. C'est dans cet esprit, qu'il s'est livré à la restauration de plusieurs monuments religieux en Polynésie française. Ses travaux sur le marae Marae Ta'ata de Tahiti demeurent exemplaires (1975), non seulement par la qualité de la reconstitution elle-même, mais aussi parce qu'ils furent précédés d'une étude minutieuse et suivis d'une publication détaillées, contrairement à bien d'autres entreprises de ce type, même récentes.

Dans le cadre de son activité professorale à l'université Paris I (Panthéon-Sorbonne), José Garanger a formé deux générations d'archéologues océanistes qu'il a fait bénéficier de son immense savoir sur la préhistoire du Pacifique mais aussi de son sens du respect des hommes. En parallèle à ses études sur le terrain, il a été très tôt impliqué dans la définition de typologies structurées et méthodiques pour mieux étudier et comprendre les anciens objets océaniens, mais également certains aménagements découverts par les archéologues. Suivant la voie tracée par André Leroi-Gourhan, ses publications sur les pilons (1967b), sur la typologie des hameçons océaniens (1965c), sur les herminettes (1972b), mais également sur les formes de sépultures (1979a), les fosses (1979b) et les foyers océaniens (1979c) continuent aujourd'hui à être référencées dans les travaux de synthèse.

Sans se départir d'une certaine humilité, il a été un vrai maître, aussi généreux que bienveillant. Sa position de leader discret l'a vu être, à partir du début des années 1970, le Secrétaire général de la Société des Océanistes, remplaçant ainsi le père O'Reilly à ce poste central pour la vie de notre Société durant plus d'une décennie. Après avoir occupé le lourd fauteuil de président de la Société durant un mandat au milieu des années 1980, José continua à occuper une place majeure dans le fonctionnement des Océanistes jusqu'au début du troisième millénaire, en étant reconduit à de nombreuses élections à un poste de vice-président. Durant toutes ces années, il contribua à maintenir la haute qualité des numéros du Journal de la Société des Océanistes et fut un moteur essentiel de la politique éditoriale de la Société.

L'influence incontournable que José Garanger a eue sur la connaissance du passé de l'Océanie et son rôle essentiel au sein de la société des Océanistes durant plus de trente ans, devait se traduire par un numéro spécial d'hommages du Journal de la Société des Océanistes. Les contributions d'auteurs proposées dans les pages qui suivent, dont la majorité n'avait pas pris part à l'ouvrage d'hommages publié en 1996, prolongent ce dernier et témoignent de résultats acquis depuis la parution du Festschrift dans des directions que José Garanger avait, souvent le premier, explorées. Cette dette intellectuelle, évidente chez les élèves de ce grand professeur qui se sont succédés durant trois décennies à ses cours, est aussi reconnue par de jeunes chercheurs qui n'eurent pas la chance de bénéficier de ses enseignements et par des collègues étrangers qui, suivant ses traces au Vanuatu ou en Polynésie, peuvent prendre la mesure de son apport déterminant à la connaissance du passé des peuples du Pacifique.

Cette contribution à multiples voix s'ouvre sur un texte de Daniel Frimigacci, qui fut, grâce aux efforts et au soutien de José, le premier archéologue à être recruté par le CNRS sur la spécificité océaniste au milieu des années 1970 . Le mode d'expression choisi, sous la forme d'une lettre, exprime de façon émouvante toute la tendresse que l'ensemble des « élèves » de José avait pour 


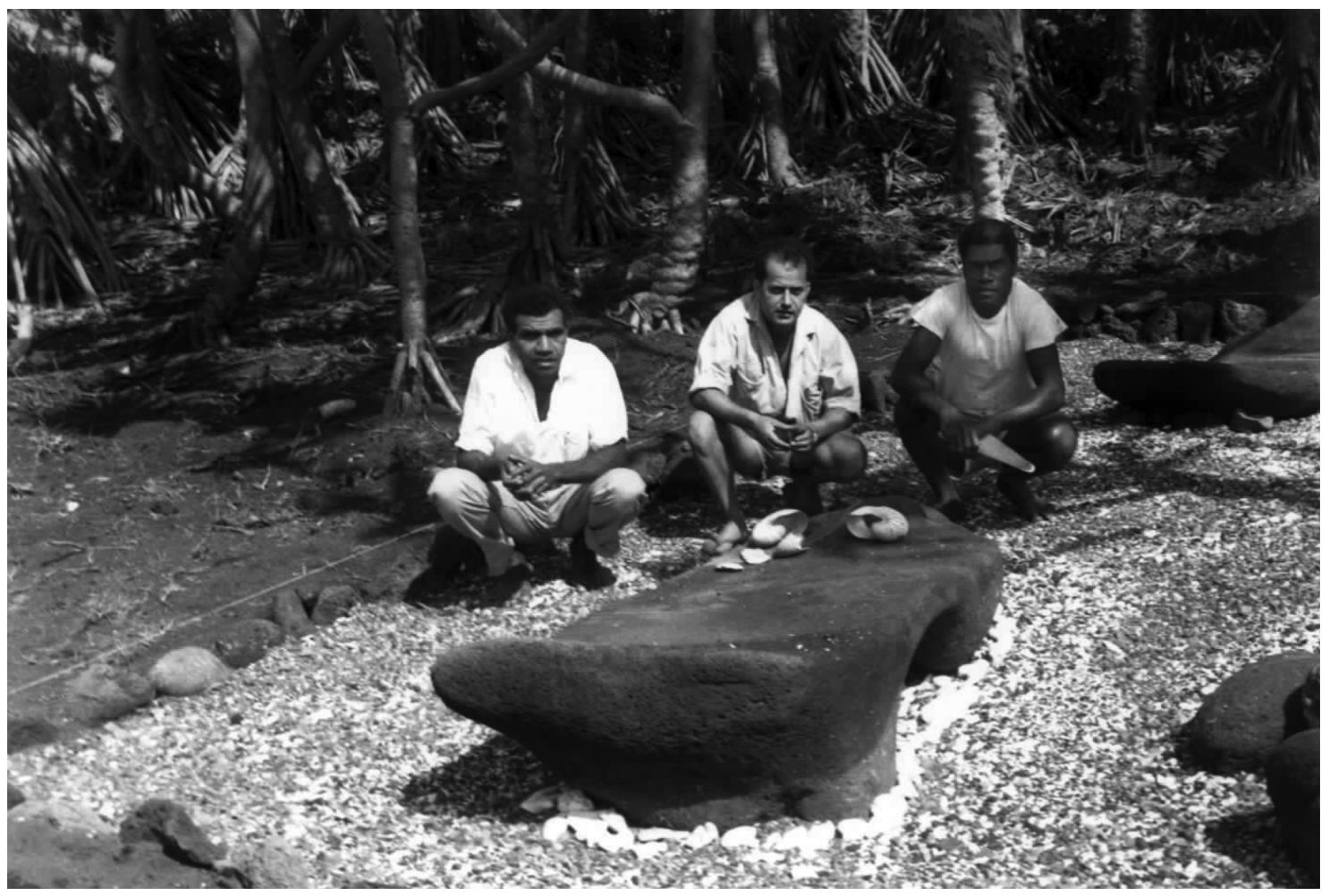

Рното 1. - José Garanger sur un site archéologique de l'île de Tongoa (Vanuatu) en 1967, entouré par deux chefs de village

ce personnage attentionné. Suit ensuite l'hommage poétique de Pierre Ottino-Garanger à ce professeur qui, pour lui, fut aussi un père.

L'attention aux autres et, en particulier, aux populations océaniennes qu'il avait côtoyées au cours de ses nombreuses missions de terrain, s'exprime également dans l'article de Christian Coiffier, la première de trois contributions sur le Vanuatu, archipel où José Garanger a longuement travaillé dans les années 1960 dans le cadre de son programme ethnoarchéologique novateur (voir 1997). L'auteur présente le processus de retour, vers leur pays d'origine, des collections archéologiques mises au jour par José Garanger. Le texte rappelle l'engagement moral qu'avait pris José envers ses hôtes mais met également en exergue les multiples intermédiaires qui ont œuvré, parfois face à des situations administratives compliquées, pour le rapatriement des objets archéologiques découverts au Vanuatu.

Stuart Bedford, dans un article synthétisant les nouvelles données sur la chronologie céramique du centre du Vanuatu (Bedford, 2006), rappelle l'importance majeure des travaux pionniers de José Garanger pour échafauder, dans les années 1960, une typologie des poteries issues de ses fouilles, à une époque où le sujet n'avait été traité quasiment nulle part ailleurs dans le Pacifique ouest (voir 1970 et 1972a : 121-128). Au vrai sens du terme, il se trouvait devant une page blanche, sans points de repères assurés pour construire une chronologie. L'article de Bedford démontre à travers des données de fouilles récentes, que l'hypothèse de la présence de deux groupes culturels différents en Mélanésie centrale durant le premier millénaire avant J.-C., l'un fabriquant des poteries incisées Mangaasi et l'autre des poteries pointillées Lapita, peut être réfutée. Ce sont les évolutions typologiques et stylistiques à partir de la tradition céramique pointillée Lapita produite sur l'île d'Efaté il y a 3000 ans, qui ont engendré au bout de quelques siècles la tradition Erueti, puis la tradition incisée et appliquée nommée Mangaasi par José Garanger.

Les évolutions culturelles de l'archipel du Vanuatu identifiées à travers la diversification des traditions céramiques, sont abordées sous l'angle des traditions funéraires dans la contribution proposée par Frédérique Valentin et ses collègues. Ce texte passe en revue les spécificités des rituels d'inhumation identifiés d'une part dans le cimetière Lapita de Téouma, d'autre part dans différents sites funéraires fouillés au cours des deux dernières décennies au Vanuatu et enfin dans les sépultures fouillées dans les années 1960 par José Garanger, avec en particulier le grand ensemble attribué au héros Roy Mata (1972a : 


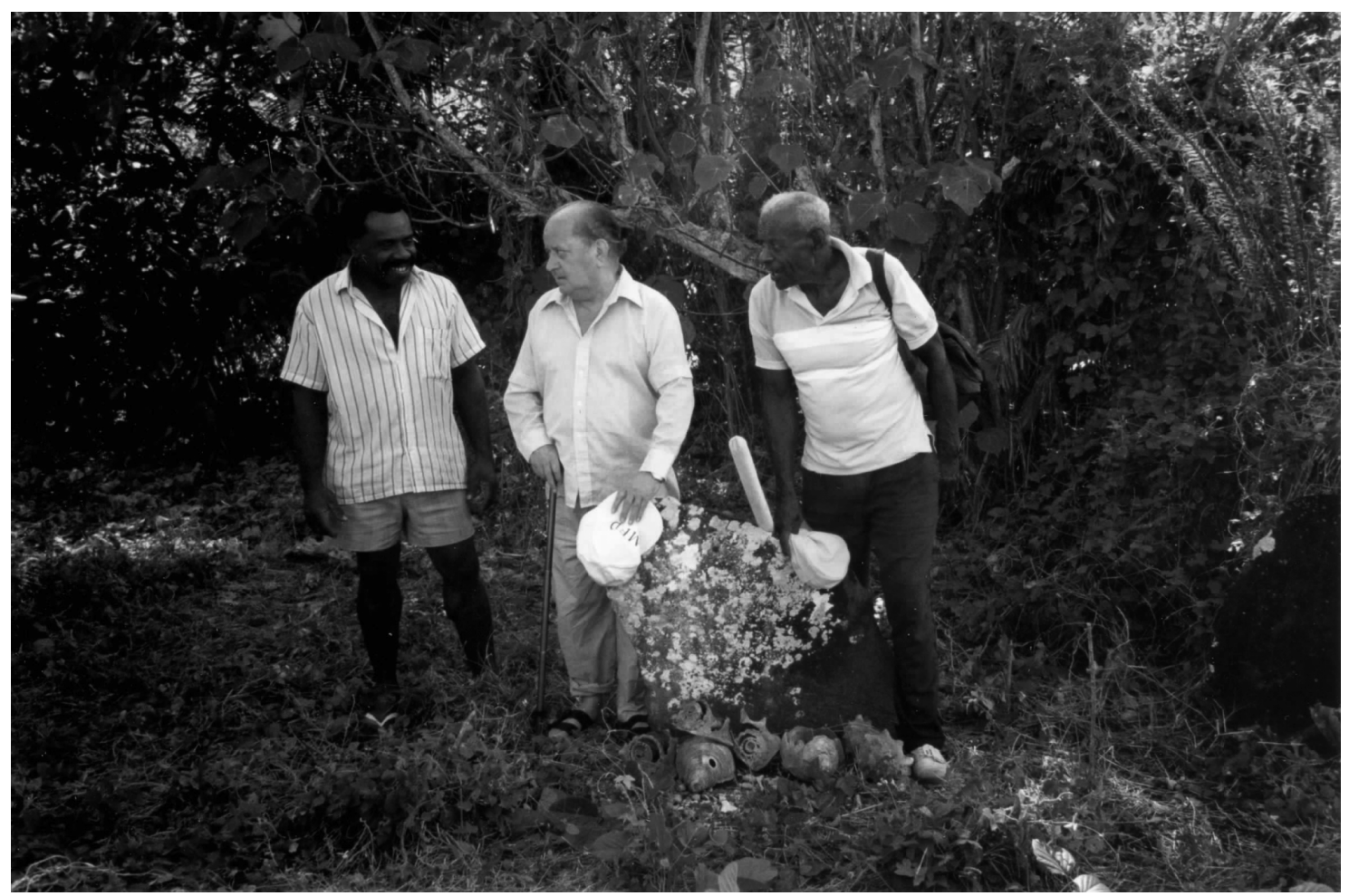

Рното 2. - José Garanger à Retoka (Vanuatu) en 1996

59-77). La synthèse des données démontre une évolution et une diversification massives des traditions d'enterrement au cours du temps dans le centre du Vanuatu. Ce résultat est à l'image de la diversité des ensembles culturels de l'archipel, posant la question de la nature des dynamiques à l'œuvre au cours des presque trois mille ans de la chronologie pré-européenne dans le centre de l'arc mélanésien.

Quittant le Vanuatu, l'article de l'équipe de Christophe Sand s'attache à souligner une autre influence léguée par José Garanger, celle de la restauration et de la mise en valeur des sites traditionnels océaniens occupés au cours des derniers siècles, dans un contexte où ses collègues anglo-saxons s'attachaient principalement à fouiller des sites très anciens, liés aux origines océaniennes. Si tout le monde connaît les travaux de restauration de marae réalisés par José Garanger en Polynésie française (1975), son intérêt pour la réhabilitation des sites l'avait déjà incité dans les années 1960 à remettre en forme, après leur fouille, certains ensembles de sépultures du Vanuatu. Le cas présenté dans l'article du département Archéologie de NouvelleCalédonie concerne un ancien site d'habitat kanak localisé dans l'intérieur de la Grande Terre calédonienne. L'introduction rappelle que, si de nombreux travaux anthropologiques ont présenté des données sur les anciennes traditions d'habitat kanak, structurées en particulier autour du symbole de l'allée centrale et de la case de haut d'allée (Boulay, 1990), il a fallu attendre les deux dernières décennies pour qu'émergent des études archéologiques focalisées sur ce type de vestige. L'étude menée sur le hameau abandonné de Tipéhéne, présentée dans cet article, est le premier programme dédié en NouvelleCalédonie entièrement à l'archéologie d'un ancien lieu d'habitat kanak. Entrant dans une démarche de valorisation du patrimoine kanak, qui est un des axes culturels centraux développés par la province Nord, le projet avait pour objectif la mise en valeur du site traditionnel, dans une perspective culturelle et touristique. Mais le projet a également inclus un volet scientifique, avec une analyse spatiale du hameau ainsi que des fouilles de surface et l'ouverture de plusieurs sondages stratigraphiques, en vue de définir une chronologie d'occupation. Ces différentes données ont permis de démontrer pour la première fois l'existence d'une chronologie progressive d'aménagement de ce type de site d'habitat kanak au cours du temps. Les résultats obtenus permettent également de souligner les nombreuses convergences entre les données des traditions orales et les résultats archéologiques obtenus. Symboliquement, le différend foncier apparu 
entre chefferies à la fin du programme, rappelle à quel point l'archéologie de terrain en Océanie ne peut faire abstraction des cadres sociaux et coutumiers contemporains.

Si l'exemple calédonien reste encore unique en son genre, la tradition de mise en valeur de sites traditionnels, débutée par José Garanger, a été appliquée à grande échelle dans les années 1990 sur l'île d'Uvea (Wallis) en Polynésie occidentale, sous l'impulsion de Daniel Frimigacci (Frimigacci et Hardy, 1997). Sous la plume de Maurice Hardy, qui fut longtemps le secrétaire du laboratoire de José Garanger, l'article suivant, dernier focalisé sur le Pacifique sud-ouest, présente un de ces sites traditionnels, mis en valeur en 1995. Il s'agit d'une plate-forme en blocs de basalte appelée Mālama Tagata, localisée en arrière du bord de mer sur la côte ouest de l'île, à proximité de la grande résidence du porteur de titre Kalāfilia, qui occupe une place importante dans l'histoire d'Uvea. Le Mālama Tagata est surtout connu grâce à une tradition orale le désignant comme le lieu de naissance d'un haut dignitaire nommé Alokuaulu, lié au royaume de Tonga. Les fouilles réalisées dans le cadre de la restauration du monument, ont permis d'identifier deux périodes successives de construction de la structure. Une première plate-forme, qui aurait été construite au $\mathrm{XII}^{\mathrm{e}}$ ou au $\mathrm{XIII}^{\mathrm{e}}$ siècle, a quelque temps plus tard été couverte par un aménagement plus important, comportant en particulier un pavage, un chemin de tour ainsi que des postes de guet. Le Mālama Tagata, interprété par Maurice Hardy comme une ancienne place de réunion, était relié aux sites importants qui l'environnaient et aux routes aménagées pour accéder au reste de l'île, par des chemins surélevés en gros blocs de basalte. Claire Moyse-Faurie propose à la suite de cet article, la transcription de la tradition orale intitulé Ko te mālama tagata, les « hommes-torches », histoire de Kalāfilia, qu'elle a recueillie sur le terrain en août 1999.

Plus à l'est du Pacifique, Claude Robineau, à partir des marae auxquels José Garanger a consacré l'essentiel de ses travaux en Polynésie française et de faits datant de la période juste postérieure aux contacts avec les Européens, remet en question la reconstitution, probablement influencée par les conceptions occidentales, de la chefferie tahitienne comme un territoire aux frontières bien délimitées pour proposer une interprétation, semble-t-il plus proche de celle des anciens Mā'ohi, d'un chaînage, un réseau de marae ari' $i$ affiliés, d'espaces réticulés comme on en retrouve ailleurs dans le Pacifique.

Rendant compte des principaux résultats obtenus lors du programme de recherches que, depuis 2001, ils conduisent dans l'archipel des Gambier (Kirch and Conte, 2004), Patrick Kirch et Éric Conte publient ici de nouvelles données permettant de dater l'arrivée de l'homme dans ces îles et de définir les grandes étapes de l'histoire de cette communauté, que de futures recherches devront encore préciser et documenter. Une reconstitution diachronique de l'exploitation humaine des milieux écologiques est également proposée qui, au fil du temps, aboutit à des situations différentes. Tandis que le milieu terrestre s'appauvrissait, avec notamment une diminution drastique de la faune aviaire et une déforestation importante, les ressources marines de l'immense lagon ne semblent guère avoir été affectées par l'action des hommes. Ce programme a également permis de nuancer l'idée communément admise selon laquelle, sur les îles hautes, les vestiges monumentaux de l'ancienne culture auraient été totalement détruits par les missionnaires, tandis que l'analyse géochimique des roches commence à montrer que les Gambier participaient à des réseaux d'échanges avec d'autres îles ou archipels, comme par exemple Pitcairn.

Michel Charleux analyse des disques de pierre qu'il a retrouvés sur l'île de Eiao aux Marquises, grand lieu d'extraction d'une pierre de qualité qui servit à la fabrication d'objets que l'on retrouve dispersés en maints endroits de Polynésie orientale, fort éloignés de leur source. Il les compare avec les 'ulu maika, utilisés par les Hawaïens pour le jeu du même nom. Cet article est aussi, comme le suivant, un hommage aux études très poussées que José Garanger consacra à certains objets océaniens afin d'aider à leur description objective lors de la définition de typologies. La contribution de Christelle Carlier et Éric Conte aborde ainsi les hameçons sur lesquels on lui doit un article essentiel (1965) ici prolongé en proposant une nouvelle procédure pour les mesurer et une méthode novatrice pour reconstituer le type et la taille des hameçons complets à partir de fragments. Ces nouvelles approches sont testées sur la riche collection provenant du site de Manihina (Ua Huka, archipel des Marquises).

\section{BIBLIOGRAPHIE}

BEDFord Stuart, 2006. Pieces of the Vanuatu Puzzle. Archaeology of the North, South and Centre, Canberra, Pandanus Books, Terra Australis 23.

Boulay Roger, 1990. La maison kanak, Paris, Parenthèses-ADCK-ORSTOM, coll. Architectures traditionnelles. 
Conte Éric and Patrick KIRCH (eds), 2004. Archaeological Investigations in the Mangareva Islands (Gambier Archipelago), French Polynesia, Berkeley, University of California, Archaeological Research Facility 62.

Frimigacci Daniel et Maurice Hardy, 1997. Des archéologues, des conquérants et des forts. Talietumu, résidence tongienne d'Uvea, Versailles, Art Lys.

GARANGER José, 1964. Recherches archéologiques dans le district de Tautira, Tahiti, Polynésie française, Journal de la Société des Océanistes 20, pp. 521.

—, 1965a. Recherches archéologiques aux NouvellesHébrides (Efate et îles Shepherd), L'Homme 6, 1, pp. $59-81$.

—, 1965b, Hameçons découverts à Rangiroa, Tuamotu occidentales, Journal de la Société des Océanistes 21, pp. 142-145.

—, 1965c, Hameçons océaniens. Éléments de typologie, Journal de la Société des Océanistes 21, pp. $127-$ 13.

—, 1967a. Notes succintes sur les recherches archéologiques effectuées dans les îles du centre des Nouvelles-Hébrides, Asian Perspectives IX, pp. 154157.

_, 1967b. Pilons océaniens, Paris, Muséum national d'histoire naturelle, catalogues du musée de l'Homme, série E, Océanie 1, 92 p.

_, 1969a. Pierres et rites sacrés du Tahiti d'autrefois, Paris, Société des Océanistes, Dossier 2.

—, 1969b. L'archéologie et les îles de la Société, Bulletin de la Société des études océaniennes 168-169, pp. 247-271.

- 1970 . Incised and applied relief pottery, its chronology and development in Eastern Melanesia, extra Areal Comparisons, Studies in Oceanic Culture History, Pacific Anthropological Records 12, pp. 53-67.

—, 1972a. Archéologie des Nouvelles-Hébrides, contribution à la connaissance des îles du centre, Paris, Société des Océanistes, Publications de la société des Océanistes 30.

-, 1972b. Herminettes lithiques océaniennes, éléments de typologie, Journal de la Société des Océanistes 28, pp. 253-274.

_, 1975. Marae Marae Ta'ata, travaux effectués par la mission archéologique ORSTOM-CNRS en 1973 et 1974. Paris, CNRS.
—, 1979a. Les sépultures en Océanie, in A. LeroiGourhan (éd.), Séminaire sur les structures d'habitat: les sépultures, Collège de France, Revista do Museu Paulista, Universidade de Sao Paulo, vol. XXVI, pp. 76-80.

—, 1979b. Fossés et fosses, caches en Océanie, in A. Leroi-Gourhan (éd.), Séminaire sur les structures d'habitat : les sépultures, Collège de France, Revista do Museu Paulista, Universidade de Sao Paulo, vol. XXVI, pp. 121-133.

_, 1979c. Types de foyers en Océanie, in A. LeroiGourhan (éd.), Séminaire sur les structures d'habitat: les sépultures, Collège de France, Revista do Museu Paulista, Universidade de Sao Paulo, vol. XXVI, pp. 29-36.

—, 1980a. Prospections archéologiques de l'îlot Fenuaino et des Vallées Aiurua et Vaiote à Tahiti, Journal de la Société des Océanistes 35, pp. 77104.

—, 1980b. Tradition orale et préhistoire en Océanie, in A. Schnapp (éd.), L'Archéologie aujourd'hui, ch. VI, Paris, Hachette.

-, 1982a. Archaeology of the New Hebrides, Sydney, Oceania Monograph 24

—, 1987a. L'archéologie océanienne, résultats et perspectives, Journal de la Société des Océanistes 41, pp. 101-102.

_, 1997. Oral traditions and archaeology: two case studies from Vanuatu, in R. Blench and M. Spriggs (eds), Archaeology and Language I. Theoretical and Methodological Orientations, London and New York, Routledge, One World Archaeology 27, pp. 321-330.

Garanger José et Anne Lavondes, 1967. Recherches archéologiques à Rangiroa, Journal de la Société des Océanistes 22, pp. 23-36.

Julien Michèle, Michel Orliac et Catherine OrliaC (éds), 1996. Mémoire de pierre, mémoire d'homme. Tradition et archéologie en Océanie, Paris, Publications de la Sorbonne.

SAND Christophe, 2003. Introduction to a conference: commemorating the first excavation at Lapita, in $\mathrm{C}$. Sand (ed.), Pacific Archaeology: assessments and prospects. Proceedings of the International Conference for the 50th anniversary of the first Lapita excavation. Koné-Nouméa 2002, Nouméa, les cahiers de l'Archéologie en Nouvelle-Calédonie 15, pp. 1-10. 